\title{
Observations and Modeling of Fog by Cloud Radar and Optical Sensors
}

\author{
Yunlong Li \\ TU Delft Climate Institute \\ Delft University of Technology \\ Delft, The Netherlands \\ Y.Li-5@tudelft.nl
}

\author{
Peter Hoogeboom \\ TU Delft Climate Institute \& TNO \\ Delft \& Den Haag, The Netherlands \\ P.Hoogeboom@tudelft.nl
}

\author{
Herman Russchenberg \\ TU Delft Climate Institute \\ Delft University of Technology \\ Delft, The Netherlands \\ H.W.J.Russchenberg@tudelft.nl
}

\begin{abstract}
Fog is a significant factor affecting the public traffic because visibility is reduced to a large extent. Therefore the determination of optical visibility in fog from radar instruments has received much interest. To observe fog with radar, high frequency bands (millimeter waves) have the best option. A $35 \mathrm{GHz}$ cloud radar at the Cabauw Experimental Site for Atmospheric Research in the Netherlands has been used to make fog measurements in the "fog mode". Meanwhile, in-situ visibility sensors (Biral SWS-100) and FSSP (Forward Scattering Spectrometer Probe) installed on a mast at Cabauw are also in use which provide visibility and drop size distribution (DSD) respectively. The synergy of remote sensing instruments at Cabauw is an essential prerequisite for fog observations. A fog case is analyzed in terms of the relations between radar reflectivity, visibility, liquid water content, and effective radius which depend on the DSD. In this paper, the modeling of fog is based on the assumption of gamma distribution as fog DSD, and is compared with the fog measurements. The results indicate the reliability of the model.
\end{abstract}

Keywords-fog; millimeteter-wave radar; optical sensors; radar reflectivity; visibility; DSD; modeling

\section{INTRODUCTION}

Fog is often regarded as very small spherical water droplets suspended near the surface with the top no higher than $200 \mathrm{~m}$. As fog droplets are very small, usually no more than $20 \mu \mathrm{m}$ radius, it is hard for traditional centimeter-wave radars to observe fog. In this context, millimeter-wave radars are well suited for fog observations, since they are more sensitive to fog droplets and the attenuation due to oxygen and water vapor is very small. Scattering and absorption of radar signals by fog can be computed using Mie scattering theory. It is found both of them depend on the radar wavelength, temperature and particle properties (size, shape, dielectric properties etc.). The scattering part tends to increase with frequency and temperature, and the absorption part increases with frequency but decreases with temperature [1].

Radar reflectivity $(Z)$ and visibility ( Vis ) define the fog intensity from two different perspectives, but they are both functions of moments of DSD. Due to the $D^{6}$ dependence of radar reflectivity and $D^{2}$ dependence of visibility ( $D$ is the drop diameter), there is a significant correlation between radar reflectivity and visibility. Since radar reflectivity and visibility can be obtained from cloud radars and in-situ visibility sensors, empirical relationships can be found from the measurements. On the other hand, radar reflectivity and visibility can be calculated from DSD provided by the FSSP, and FSSP-based $\mathrm{Z}$-Vis relations can also be found. Moreover, LWC (liquid water content) has been linked with radar reflectivity and visibility, and different Z-LWC, Vis-LWC relations refer to different cloud types [2]. One of mostly used parameters in cloud studies is the effective radius $r_{e}$ defined as the ratio of the third to the second moment of DSD. Since radar reflectivity and visibility are correlated with the DSD, the effective radius can be also linked with radar reflectivity and visibility [3].

DSD is the key factor in determining the relations between radar reflectivity, visibility, liquid water content and effective radius. It is hard to find a centralized relation since DSD varies with time and space. The gamma distribution is often used to represent the DSD of fog, and the modeling of fog can be achieved on the grounds of the interactions among the macroand micro-physical parameters of fog.

In this paper, the measurements from a $35 \mathrm{GHz}$ cloud radar, in-situ visibility sensors and FSSP at Cabauw are deployed to retrieve the interactions among the macro- and micro-physical parameters of fog for the modeling of fog. Section II describes the measurement setup in the "fog mode". Section III evaluates the cloud radar sensitivity to fog. Section IV analyzes the observations of a fog event and introduces the modeling of fog. The model results are compared with the measurements. Section $\mathrm{V}$ is the conclusion and presents the outlook for future work.

\section{MEASUREMENT SETUP}

At the experimental site of Cabauw a $35 \mathrm{GHz}$ cloud radar, in-situ visibility sensors and FSSP are deployed for fog observations. In this section, the in-situ and remote sensing instruments especially the cloud radar working in the "fog mode" are briefly described.

\section{A. 35 GHz Cloud Radar in the "Fog Mode"}

As the radar normally operates in zenith mode, it would yield no information about fog, because the first range gate of the radar starts at a distance of $250 \mathrm{~m}$, and the height of fog is normally less than $200 \mathrm{~m}$ in the Netherlands. Therefore, a light- 
weight aluminum reflector can be placed above the antenna of the radar during fog episodes. The reflector deflects the radar beam in a nearly horizontal direction but slightly upward at an angle of $3.5^{\circ}$ which enables to detect the fog layers at a minimum vertical height of $15 \mathrm{~m}\left(250 \mathrm{~m} \times \sin 3.5^{\circ}\right)$. However, this mode of operation will suffer from ground clutter due to the small angle. Fig. 1 shows the $35 \mathrm{GHz}$ cloud radar being prepared to operate in the "fog mode", and Fig. 2 shows the layout of radar measurements during fog episodes.

\section{B. In-situ Visibility Sensors}

The $213 \mathrm{~m}$ mast at Cabauw has been equipped with Biral SWS-100 sensors at 2, 10, 20, 40, 80, 140, $200 \mathrm{~m}$ height with visibility resolution of $10 \mathrm{~m}$ and accuracy of $10 \%$. Fig. 3 shows the Biral SWS-100 sensors on the mast.

\section{FSSP}

FSSP (Forward Scattering Spectrometer Probe) was installed at $60 \mathrm{~m}$ height of the Cabauw mast which measures the sizes and numbers of particles in the size range of $3-46.5$ $\mu m$ diameter. The size range is divided into 30 size bins with each $1.5 \mu \mathrm{m}$. Fig. 4 shows the FSSP installed at $60 \mathrm{~m}$ height of the Cabauw mast.

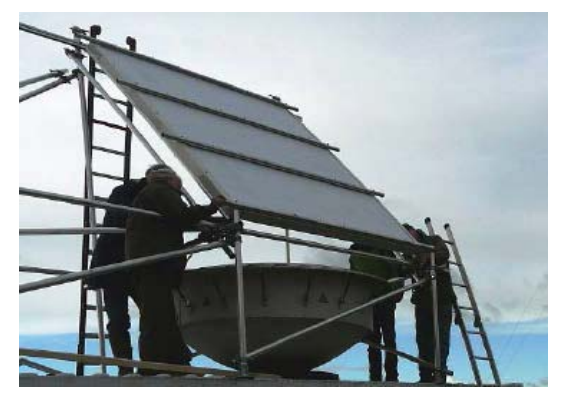

Fig. 1. The $35 \mathrm{GHz}$ cloud radar at Cabauw is prepared for the "fog mode".

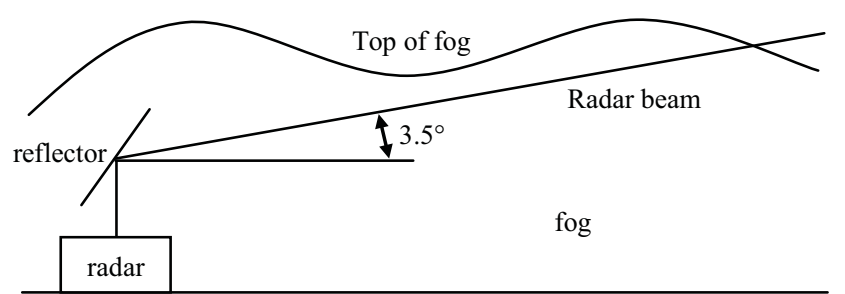

Fig. 2. The layout of radar measurements during fog episodes.

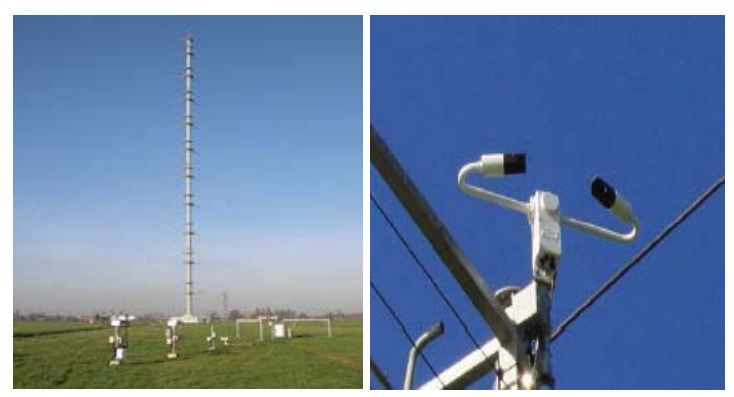

Fig. 3. The Biral SWS-100 sensors on the mast of Cabauw.

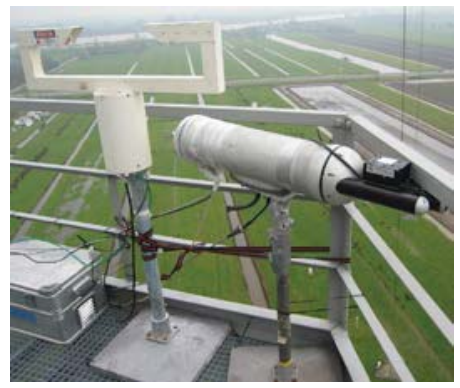

Fig. 4. The FSSP on the mast of Cabauw.

TABLE I. OPERATIONAL PARAMETERS OF THE 35 GHZ CLOUd RADAR IN THE FOG MODE.

\begin{tabular}{|c|c|}
\hline Radar Frequency & $35 \mathrm{GHz}$ \\
\hline Transmit Power & $200 \mathrm{~W}$ (maximum) \\
\hline Antenna Gain & $52 \mathrm{dBi} \pm 1 \mathrm{~dB}$ \\
\hline Pulse Length & $600 \mathrm{~ns}$ \\
\hline Noise Figure & $6.3 \mathrm{~dB}$ \\
\hline Minimum Range & $250 \mathrm{~m}$ \\
\hline Maximum Range & $3353 \mathrm{~m}$ \\
\hline Radar Beam Elevation & $3.5^{\circ}$ \\
\hline
\end{tabular}

\section{CLOUD RADAR SENSITIVITY TO FOG}

It is necessary to evaluate the cloud radar sensitivity to fog which provides a prerequisite for fog observations. Table I lists the operational parameters of the $35 \mathrm{GHz}$ cloud radar in the "fog mode".

Meteorological radar equation can be expressed when the particles are much smaller than the radar wavelength as [4]:

$$
P_{r}=\frac{\pi^{3} P_{t} G^{2} \theta \phi h|K|^{2} Z}{1024 \ln 2 \lambda^{2} R^{2} L_{s}}
$$

where $P_{r}$ is received power; $P_{t}$ is transmit power; $G$ is antenna gain; $\theta$ and $\phi$ are $3 d B$ beamwidth; $h$ is pulse length; $Z$ is radar reflectivity; $\lambda$ is wavelength; $R$ is the range from radar to target volume; $L_{s}$ is radar system loss. $K$ is the parameter related to the complex index of refraction, which is given by $K=\left(m^{2}-1\right) /\left(m^{2}+2\right), m$ is the complex index of refraction given by $m=n+i k, n$ is the index of refraction, $k$ is the absorption coefficient. $K$ is a factor depending on the radar wavelength, temperature and particle properties (size, shape, dielectric properties etc.).

The atmospheric attenuation is an important factor that has to be taken into account for millimeter-wave radars. Unlike system loss, the attenuation loss depends on the range,

$$
L_{a}(r)=k_{a} \cdot 2 R \quad(d B)
$$

where $L_{a}$ is attenuation loss in $d B ; k_{a}$ is attenuation coefficient in $\mathrm{dB} / \mathrm{km} ; R$ is the range, factor 2 illustrates the roundtrip between radar and target volume. 
The attenuation coefficient $k_{a}$ is given by the cloud attenuation model in Rayleigh approximation [4],

$$
k_{a}=\left[0.4343 \frac{6 \pi}{\lambda} \operatorname{Im}(-K)\right] \cdot L W C \quad(\mathrm{~dB} / \mathrm{km})
$$

where $L W C$ is liquid water content in $\mathrm{g} / \mathrm{cm}^{3}$.

Therefore the meteorological radar equation can be modified as:

$$
P_{r}=\frac{C|K|^{2} Z}{R^{2} \cdot\left(k_{a} \cdot 2 R\right)}
$$

where $C$ is radar constant.

The radar sensitivity can be evaluated as the SNR (Signal to Noise Ratio) set to $0 d B$ that the received power is equivalent to the noise power. Fig. 5 shows the attenuation coefficient with $L W C$ of $0.1 \mathrm{~g} / \mathrm{cm}^{3}$, and Fig. 6 shows the $35 \mathrm{GHz}$ cloud radar sensitivity to fog.

\section{FOG OBSERVATIONS AND MODELING OF FOG}

\section{A. Fog Observations}

A fog event that occurred during 17 and 18 February 2012 was observed with the described instruments at Cabauw. The fog originated from low clouds consistently lowering to the ground. Visibility began to reduce below $1 \mathrm{~km}$ at 22:27:00 UTC 2-17-2012 till 03:43:00 UTC 2-18-2012 when the clouds lifted. All the instruments including the $35 \mathrm{GHz}$ cloud radar, in-situ visibility sensors and FSSP worked well when fog happened.

Fig. 7 depicts the evolution of the fog starting from the night before and dissipating in the next early morning, and Fig. 8 shows the observations during fog episodes. As the FSSP is installed at $60 \mathrm{~m}$ height of the Cabauw mast, the visibility measurements from the in-situ visibility sensor installed at 40 $\mathrm{m}$ of the mast are used for comparison. It is seen from the observations that during fog episodes the visibility from the insitu visibility sensor matches quite well with the one derived from the FSSP, but there is at least one order of magnitude difference between them beyond fog episodes. The similar situation occurred to the radar reflectivity, but it has to be noted that many data in the radar reflectivity measurements are "lost" due to the cloud mask processing.

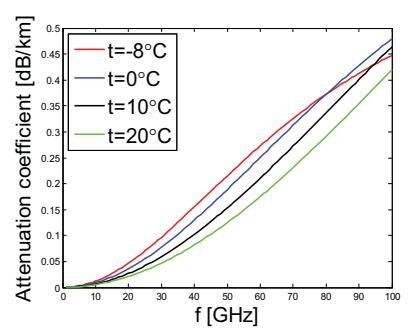

Fig. 5. Attenuation coefficient with LWC of $0.1 \mathrm{~g} / \mathrm{cm}^{3}$.

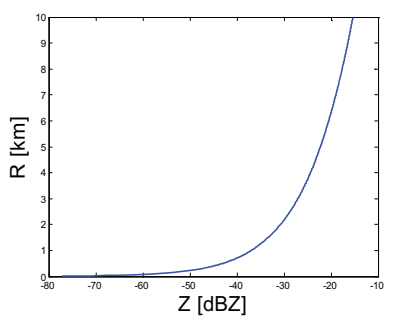

Fig. 6. Evaluation of the $35 \mathrm{GHz}$ cloud radar sensitivity to fog.
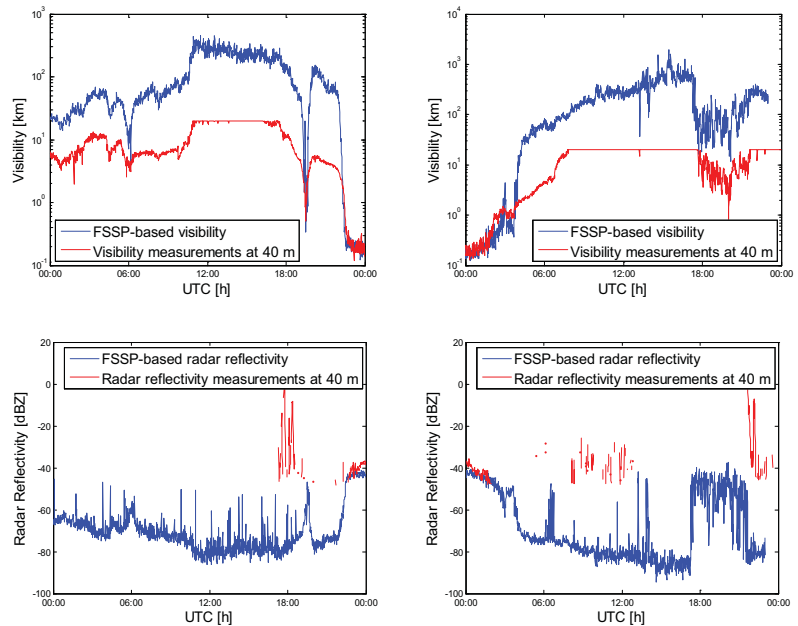

Fig. 7. The evolution of the fog on 17 and 18 February 2012 at Cabauw. Left panels are on 17 February, and right panels are on 18 February.
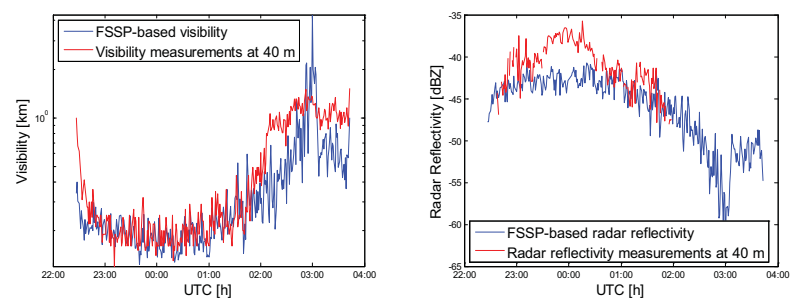

Fig. 8. The observations during fog episodes of 17 and 18 February 2012.

\section{B. Modeling of Fog}

The gamma distribution is often used to represent the DSD of fog as [3]:

$$
n(D)=\frac{N_{0}}{D_{m}^{v} \cdot \Gamma(v)} \cdot D^{v-1} \cdot e^{-\frac{D}{D_{m}}}, D \geq 0
$$

where $N_{0}$ is number concentration; $\Gamma(v)$ is gamma function; $v$ and $D_{m}$ are the shape and scale parameters of gamma distribution.

$Z, V i s, L W C$ and $r_{e}$ are all functions of moments of DSD, they are represented as the sum of particle numbers in each size bin $i$ :

$$
\begin{gathered}
Z=64 \sum_{i} N_{i} r_{i}^{6} \\
V i s=\frac{-\ln \epsilon}{2 \pi \sum_{i} N_{i} r_{i}^{2}}
\end{gathered}
$$

where $\epsilon$ is the threshold of contrast and normally equals to 0.05 .

$$
L W C=\frac{4 \pi}{3} \rho_{w} \sum_{i} N_{i} r_{i}^{3}
$$




$$
r_{e}=\frac{\sum_{i} N_{i} r_{i}^{3}}{\sum_{i} N_{i} r_{i}^{2}}
$$

The modeling of fog can be achieved from the interactions among the DSD-based parameters, and validated by the measurements.

$$
\begin{gathered}
V i s \cdot Z=\frac{-\ln \epsilon}{2 \pi / 4} \cdot D_{m}^{4} \cdot \frac{\Gamma(v+6)}{\Gamma(v+2)} \cdot 10^{-9} \\
V i s \cdot L W C=\frac{-\ln \epsilon}{2 \pi} \cdot \frac{4 \pi}{3} \rho_{w} \cdot r_{e} \cdot 10^{-9} \\
V i s \cdot Z=\frac{-64 \cdot \ln \epsilon}{2 \pi} \cdot \frac{(v+3)(v+4)(v+5)}{(v+2)^{3}} \cdot r_{e}^{4} \cdot 10^{-9}
\end{gathered}
$$

where Vis is in $\mathrm{km} ; Z$ is in $\mathrm{mm}^{6} / \mathrm{m}^{3} ; L W C$ is in $\mathrm{g} / \mathrm{cm}^{3}$; $r_{e}$ is in $\mu m$.

Fig. 9, Fig. 10, Fig. 11 show the observations of the described fog event, and the modeling of the $V i s-Z$, $V i s \cdot L W C-r_{e}, V i s \cdot Z-r_{e}$ relations. In Fig. 9, $v$ and $D_{m}$ are adopted as 2 and 1.2. As seen from the observations, $V i s-Z$ pertains to a power law as $V i s=a \cdot Z^{b}$, and the fitting parameters are $a=0.018, b=-0.262$ for the radar and visibility sensor measurements, $a=3.5186 \cdot 10^{-4}, b=-0.6325$ for the FSSP-based measurements. In Fig. 10, Vis $\cdot L W C-r_{e}$ pertains to a linear relation as $V i s \cdot L W C=C \cdot r_{e}$, and $C=1.888 \cdot 10^{-9}$ for the FSSP-based measurements, $C=1.997 \cdot 10^{-9}$ for the modeling. In Fig. $11, \mathrm{Vis} \cdot Z-r_{e}$ pertains to a power law as $V i s \cdot Z=a \cdot r_{e}^{b}$, and therefore $\log _{10}(\mathrm{Vis} \cdot \mathrm{Z})-\log _{10} r_{e}$ pertains to a linear relation as $\log _{10}($ Vis $\cdot Z)=\log _{10} a+b \cdot \log _{10} r_{e}$. The factor $a$ depends on the shape parameter of gamma distribution $v$, and $b$ should be 4 theoretically. Fitting to the FSSP-based measurements, $a$ is 3.4 which is very close to 4 . Two theoretical relations with extreme values of shape parameter $v(v=\infty$ corresponds to the $\delta$-function-like gamma distribution; $v=1$ corresponds to the exponential distribution) are also presented in Fig. 11.

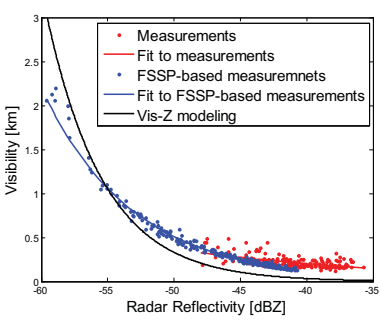

Fig. 9. Observations and modeling of Vis $-Z$ relation.

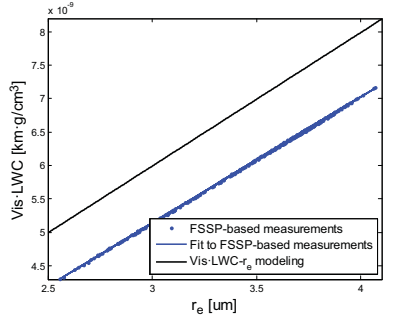

Fig. 10. Observations and modeling of $V i s \cdot L W C-r_{e}$ relation.

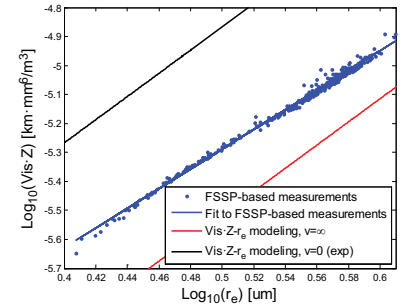

Fig. 11. Observations and modeling of $\mathrm{Vis} \cdot \mathrm{Z}-r_{e}$ relation.

\section{CONCLUSIONS}

This paper discusses the possibility of extracting optical visibility in fog from measurements with the $35 \mathrm{GHz}$ cloud radar at Cabauw. The radar sensitivity to fog was evaluated and the attenuation due to fog has to be taken into account. Meanwhile, some other remote sensing instruments (in-situ visibility sensors, FSSP) were also used to observe fog. A fog event was mainly depicted with the evolution of visibility and radar reflectivity. It is noteworthy that during fog episodes the measurements from the radar and visibility sensor match quite well with those derived from the FSSP, but there is at least one order of magnitude difference between them beyond fog episodes. The main reason was illustrated in [1]. The interactions ( $\mathrm{Vis}-Z, \mathrm{Vis} \cdot L W C-r_{e}, \mathrm{Vis} \cdot Z-r_{e}$ relation) among the macro- and micro-physical parameters of fog were retrieved based on an assumption of fog DSD for the modeling of fog, and are compared with the measurements. The results indicate the reliability of the model. However, more fog events will be analyzed in the future to validate the model.

\section{ACKNOWLEDGMENT}

The authors show great appreciation to Henk Klein Baltink and Reinout Boers (KNMI, Royal Netherlands Meteorological Institute) for providing the valuable data and open ideas.

\section{REFERENCES}

[1] Y. Li, P. Hoogeboom, and H.W.J. Russchenberg, "Radar observations and modeling of fog at $35 \mathrm{GHz}$," in $8^{\text {th }}$ European Conference on Antennas and Propogation (EUCAP), Den Haag, April 2014.

[2] A. Khain, et al, "Combined observational and model investigations of the Z-LWC relationship in stratocumulous clouds," in J. Appl. Meteor. Climatol., vol. 47, pp. 591-606, February 2008.

[3] O.A. Krasnov and H.W.J. Russchenberg, "The relation between the radar to lidar ratio and the effective radius of droplets in water clouds: an analysis of statistical models and observed drop size distributions," in $11^{\text {th }}$ Conf. on Clouds Physics, Ogden, UT, Amer. Meteor. Soc., P2.18.

[4] S. Raghavan, "Radar meteorology," Kluwer Academic Press, 2003. 